\title{
Prevalence of comorbid retinal disease in patients with glaucoma at an academic medical center
}

\author{
This article was published in the following Dove Press journal: \\ Clinical Ophthalmology \\ 13 July 2015 \\ Number of times this article has been viewed
}

\author{
Joseph F Griffith' \\ Jeffrey L Goldberg ${ }^{2}$ \\ 'Cole Eye Institute, Cleveland Clinic \\ Foundation, Cleveland, $\mathrm{OH},{ }^{2}$ Shiley Eye \\ Center, University of California San \\ Diego, La Jolla, CA, USA
}

Background: Patients with various retinal diseases and patients who have undergone retinal procedures and surgeries have an increased risk of developing ocular hypertension and glaucoma. Little is known about the epidemiology of comorbid retinal diseases in glaucoma patients. This study evaluated the prevalence of retinal comorbidities in a population of patients with five types of glaucoma.

Methods: A longitudinal, retrospective study was conducted using International Classification of Disease (ICD-9) billing records from 2003 to 2010 at an academic medical center. Patients were classified as having primary open-angle glaucoma (POAG), low tension open-angle glaucoma (NTG), pigmentary open-angle glaucoma, chronic-angle closure glaucoma (CACG), or pseudoexfoliation glaucoma (PXG) if they had at least three clinic visits with the same ICD-9 code. Patients were classified as having a retinal comorbidity if they had two visits with the same code. Variables were analyzed with the independent $t$-test, $\chi^{2}$ test, analysis of variance, or Fisher's exact test.

Results: A total of 5,154 patients had glaucoma, and $14.8 \%$ of these had a retinal comorbidity. The prevalence of comorbid retinal disease was higher in patients with POAG $(15.7 \%)$ than in those with NTG (10.7\%), PXG (10.1\%), or pigmentary open-angle glaucoma $(3.7 \% ; P<0.05)$. Two hundred and two patients had diabetic retinopathy, with POAG patients $(4.5 \%)$ having a higher prevalence than those with CACG $(1.4 \%)$ or PXG $(0.6 \% ; P<0.001)$. There were 297 patients who had macular degeneration and both POAG $(2.0 \%)$ and PXG patients $(2.9 \%)$ had a higher prevalence of nonexudative macular degeneration than those with CACG $(0 \% ; P<0.01)$. Patients with comorbid retinal disease had a higher prevalence of blindness and low vision than those without comorbid retinal disease ( $1.97 \%$ versus $1.02 \%, P=0.02)$.

Conclusion: The high prevalence of comorbid retinal disease and the nearly twofold increase in blindness and low vision in this population demonstrate the need for ophthalmologists to determine if patients have multiple etiologies for their vision loss. The higher prevalence of certain retinal diseases in POAG patients may reflect common pathophysiological processes that warrant further investigation.

Keywords: glaucoma frequency, retina frequency, comorbid retinal disease, glaucoma prevalence, retina prevalence

\section{Introduction}

Glaucoma and various retinal diseases are leading causes of vision loss worldwide. Numerous retinal diseases are associated with and lead to various types of glaucoma. Ischemic conditions such as central retinal vein occlusion (CRVO) or branch retinal vein occlusion (BRVO), central or branch retinal artery occlusion, malignancy, and proliferative diabetic retinopathy are associated with neovascular glaucoma. ${ }^{1}$ Various types of uveitis, ${ }^{2-5}$ including Behçet's disease,${ }^{6}$ sarcoidosis, ${ }^{4}$ syphilis, ${ }^{4}$ Fuchs iridocyclitis, ${ }^{4}$ and juvenile rheumatoid arthritis ${ }^{4}$ are causes of secondary glaucoma.
Correspondence: Joseph F Griffith

Cole Eye Institute, 9500 Euclid Avenue,

i- I3, Cleveland, OH 44I06, USA

$\mathrm{Tel}+\mathrm{I} 2023655778$

Fax +I 2164452226

Email griffij2@ccf.org submit your manuscript | www.dovepress.com

Dovepress

http://dx.doi.org//0.2147/OPTH.S8585 (c) (7) (5) 2015 Griffith and Goldberg. This work is published by Dove Medical Press Limited, and licensed under Creative Commons Attribution - Non Commercial (unported, v3.0) License. The full terms of the License are available at http://creativecommons.org/licenses/by-nc/3.0/. Non-commercial uses of the work are permitted without any further issions beyond the scope of the License are administered by Dove Medical Press Limited. Information on how to request permission may be found at: http://www.dovepress.com/permissions.php 
Retinitis pigmentosa is associated with primary open-angle and primary-angle closure glaucoma. ${ }^{7}$ Patients with retinal detachments have higher rates of both ocular hypertension and glaucoma than people in the general public. ${ }^{8}$

Patients undergoing retinal procedures and retinal surgeries are at increased risk for elevated intraocular pressure (IOP). Intravitreal steroid injections, especially intravitreal triamcinolone acetonide, are associated with elevations in IOP in approximately $50 \%$ of patients, which can require treatment with topical medications and, occasionally, glaucoma surgery. ${ }^{9,10}$ Panretinal photocoagulation is associated with elevated $\mathrm{IOP}^{11}$ and acute-angle closure glaucoma. ${ }^{12,13}$ Studies on intravitreal anti-vascular endothelial growth factor injections show an association with sustained IOP elevation ${ }^{14}$ requiring topical medications and, occasionally, glaucoma surgery. ${ }^{15}$ Vitreoretinal surgeries ${ }^{16}$ including both simple vitrectomy ${ }^{17}$ (eg, vitrectomy without gas, scleral buckle, or silicon oil) and complex vitrectomy, ${ }^{17,18}$ are associated with IOP elevation during the first 24 hours after the operation. In addition, vitrectomy with silicon oil ${ }^{19}$ and vitrectomy without silicon oil ${ }^{20,21}$ are both associated with the development of glaucoma.

Studies have documented the risk of elevated IOP and glaucoma in patients with certain retinal diseases and in certain postoperative or post-procedure retina patients. Little is known about the prevalence of comorbid retinal disease in patients with glaucoma. The prevalence of comorbid retinal diseases may help clinicians consider whether a patient's vision loss is due to glaucoma or whether it is multifactorial with other disease processes involved. This study examines the prevalence of various types of retinal disease in a group of patients with five different types of glaucoma at a single academic medical center.

\section{Materials and methods}

A longitudinal, retrospective study was conducted at a single academic medical center using International Classification of Disease (ICD-9) and Current Procedure Terminology billing records from June 1, 2003 to November 30, 2010. All patients were aged 40 years or older at the time of their last clinical examination and were classified using ICD-9 billing records as having primary open-angle glaucoma (POAG [365.11]), low tension open-angle glaucoma (NTG [365.12]), pigmentary open-angle glaucoma (365.13), chronic-angle closure glaucoma (CACG [365.23]), or pseudoexfoliation glaucoma (PXG [365.52]). This study did not include patients who were glaucoma suspects (365.0-365.04) in order to focus on patients with known glaucomatous damage. Patients with neovascular glaucoma (365.63) were excluded because there are well-established associations between neovascular glaucoma and diabetic retinopathy, CRVO, various ocular malignancies, and ocular ischemic syndrome. ${ }^{22}$ Patients were similarly classified as having a retinal comorbidity based on ICD-9 codes for retinal detachments and defects (361.0-361.9), other retinal disorders (362.0-362.9), or chorioretinal inflammation, scars, and other disorders of the choroid (363.0-363.9). The Human Subject Research Office of University of Miami Institutional Review Boards approved this study. The study was conducted in accordance with the Declaration of Helsinki.

Patients were included in the study if they had had at least three clinical examinations with the same glaucoma diagnosis ICD-9 code, and had received follow-up care in ophthalmology clinics over at least 2 years. Each patient was categorized as having one type of glaucoma to ensure that each glaucoma subset was composed of unique patients. Patients were excluded if they met the criteria for more than one glaucoma diagnosis (at least three clinical examinations over 2 years for each glaucoma diagnosis).

Patients were categorized as having a retinal comorbidity if they had at least two visits on different dates with the same retinal ICD-9 code. We did not differentiate the type of visit (clinical visit, ophthalmology emergency room visit, surgery visit, or procedure visit). Patients were categorized as having blindness and low vision (ICD-9 369) if they had two visits with ICD-9 code 369 . Unlike with the comorbid retina population, we did not require patients to have two visits associated with the same specific (two decimal place) ICD-9 code for blindness and low vision.

Some vitreoretinal ICD-9 codes were excluded because a preliminary analysis revealed that there were no patients with any of the following diagnoses: vitreous abscess (360.04), pan uveitis (360.12), or papilledema (377.00-377.04). Four patients with optic neuritis (377.3), eleven patients with optic atrophy (377.1), and eleven patients with other disorders of the optic nerve (377.4) were similarly excluded.

Statistical analysis was performed using JMP version 10 (SAS Institute Inc, Cary, NC, USA). Categorical variables were analyzed with the $\chi^{2}$ test or Fisher's exact test. Continuous variables were analyzed with the independent $t$-test, analysis of variance (ANOVA), or least significant difference post hoc $t$-test.

\section{Results}

A total of 5,154 patients met the criteria for having glaucoma, with the majority of these patients having POAG $(n=4,171$, 
$80.9 \%)$ and 2,142 (41.6\%) seen for at least 5 years. Seven hundred and sixty of these glaucoma patients had a retinal diagnosis, and 35 of these had multiple categories of retinal diagnoses (eg, both a diagnosis within retinal detachments and also within other retinal disorders). Overall, the prevalence of comorbid retinal disease in our glaucoma patient population was $14.8 \%(760 / 5,154)$. The prevalence of retinal detachment was $0.99 \%$ (ICD-9 361.xx, 51/5,154), the prevalence of other retinal disorder was 13.3\% (ICD-9 362.xx, $684 / 5,154)$, the prevalence of other disorders of the choroid was $0.33 \%$ (ICD-9 363.xx, 17/5,154), and the prevalence of disorders of the vitreous body was $0.83 \%$ (ICD-9 379.2x, 43/5,154).

Characteristics of glaucoma patients with and without retinal comorbidities are listed in Table 1. Overall, there were more females $(2,906,56.3 \%)$ than males in this study $(2,248,43.7 \%)$. There was no difference in rates of comorbid retinal disease between males and females $(P=0.29$, $\chi^{2}$ test). Glaucoma patients with a comorbid retinal disease were older than glaucoma patients without a retinal disease (mean age 75.4 years versus 73.1 years, $P<0.0001$, independent $t$-test). Of patients with retinal comorbidities, PXG patients were older than those with POAG, NTG, or CACG (mean age 83.1 years versus 73.7-75.2 years, $P<0.05$, ANOVA and least significant difference post hoc $t$-tests). There was no difference in mean age between patients with PXG and those with pigmentary open-angle glaucoma (83.1 years versus 73.8 years, $P>0.05$, Fisher's exact test). There were no differences in mean age between POAG, NTG, pigmentary open-angle glaucoma, and CACG patients with regard to retinal comorbidities ( $P>0.05$, ANOVA).

We next asked what types of glaucoma patients are most likely to have comorbid retinal disease. Table 2 and Figure 1

Table I Age and sex of patients with and without comorbid retinal disease

\begin{tabular}{|c|c|c|}
\hline & $\begin{array}{l}\text { Glaucoma patients } \\
\text { with } R C(n=760)\end{array}$ & $\begin{array}{l}\text { Glaucoma patients } \\
\text { without } R C(n=4,394)\end{array}$ \\
\hline \multicolumn{3}{|c|}{ Age, years (\%)* } \\
\hline $40-49$ & $16(2.1)$ & $160(3.6)$ \\
\hline $50-59$ & $65(8.6)$ & $48 \mid(10.9)$ \\
\hline $60-69$ & $|6|(2 \mid .2)$ & $1,013(23.1)$ \\
\hline 70-79 & $221(29.1)$ & $\mathrm{I}, 334(30.4)$ \\
\hline $80-89$ & $229(30.1)$ & I,I $27(25.6)$ \\
\hline$\geq 90$ & $68(8.9)$ & $279(6.3)$ \\
\hline Mean & $75.4 \pm 11.5$ & $73.1 \pm 11.8$ \\
\hline \multicolumn{3}{|l|}{ Sex, n (\%) } \\
\hline Female & $419(55.1)$ & 2,487 (56.6) \\
\hline Male & $34 \mid(44.9)$ & I,907 (43.4) \\
\hline
\end{tabular}

Note: *Age as of last visit.

Abbreviation: RC, retinal comorbidity. list the proportions of patients with comorbid retinal disease by glaucoma type. There was a larger proportion of POAG patients with comorbid retinal disease (15.7\%) than NTG patients $\left(10.7 \%, P=0.014, \chi^{2}\right.$ test), PXG patients $(10.1 \%$, $P=0.008, \chi^{2}$ test $)$, and pigmentary open-angle glaucoma patients $(3.7 \%, P=0.013$, Fisher's exact test). There were no other differences in the proportions of comorbid retinal disease between type of glaucoma patients ( $P>0.05, \chi^{2}$ test and Fisher's exact test).

We next examined what types of retinal disease are most common in glaucoma patients. Tables 3-6 list the numbers of patients with each type of diagnosis. The most common diagnoses included cystoid macular degeneration (125), nonexudative senile macular degeneration (94), unspecified senile macular degeneration (89), background diabetic retinopathy (82), and proliferative diabetic retinopathy (78), as shown in Figure 2.

We then asked whether there is a relationship between glaucoma subtype and diabetic retinopathy, age-related macular degeneration (AMD), and retinal vascular occlusions, which are three of the most common retinal comorbid diagnoses. Two hundred and thirty-one glaucoma patients had diabetes mellitus with ophthalmic manifestations (ICD-9 250.5-250.53, 362.01-362.07), of which 202 had diabetes-related retinal disease (ICD-9 362.01-362.07). The prevalence of diabetes-related retinal disease among glaucoma patients in this study was $3.92 \%(202 / 5,154)$. Fifty-six patients had visits for just proliferative diabetic retinopathy and 139 patients had visits for nonproliferative or background diabetic retinopathy. Twenty-two had visits for both of these categories. POAG patients had a higher prevalence of comorbid diabetic retinopathy (4.5\%) than CACG patients (1.4\%, $P=0.013$, Fisher's exact test) or PXG patients ( $0.6 \%$, $P<0.001$, Fisher's exact test). There was no difference in mean age between POAG, CACG, and PXG patients with diabetic retinopathy $(P>0.05$, ANOVA).

One hundred and twenty-eight glaucoma patients were diagnosed with a retinal vascular occlusion, with four patients having a central or branch artery occlusion, 124 patients having a CRVO or BRVO, and two patients having both an arterial and venous occlusion. There was no difference in the prevalence of retinal vascular occlusions according to type of glaucoma $(0 \%-2.9 \%, P>0.05$, Fisher's exact test). Similarly, there was no difference in prevalence of retinal vein occlusion according to type of glaucoma $(0 \%-2.9 \%$, $P>0.05$, Fisher's exact test).

A total of 297 glaucoma patients were diagnosed with macular degeneration (exudative, nonexudative, unspecified, 
Table 2 Comorbid retina prevalence by glaucoma type

\begin{tabular}{llllll}
\hline & POAG $(\mathbf{n}=\mathbf{4}, \mathbf{I}$ 7I $)$ & NTG $(\mathbf{n}=\mathbf{3 3 6})$ & PG $(\mathbf{n}=\mathbf{5 4})$ & CACG $(\mathbf{n}=\mathbf{2 7 7})$ & PXG $(\mathbf{n}=\mathbf{3}$ I6) \\
\hline Patients with RC & $656(15.7 \%)$ & $36(10.7 \%)$ & $2(3.7 \%)$ & $34(12.3 \%)$ & $32(10.1 \%)$ \\
Patients without RC & $3,515(84.3 \%)$ & $300(89.3 \%)$ & $52(96.3 \%)$ & $243(87.7 \%)$ & $284(89.9 \%)$ \\
\hline
\end{tabular}

Note: Proportion of POAG patients with RC exceeds those with NTG, PG, and PXG $\left(P<0.05\right.$, Fisher's exact test and $\chi^{2}$ test).

Abbreviations: POAG, primary open-angle glaucoma; NTG, low tension open-angle glaucoma; PG, pigmentary open-angle glaucoma; CACG, chronic angle-closure glaucoma; PXG, pseudoexfoliation glaucoma; RC, retinal comorbidity.

or cystoid macular degeneration), with 84 patients having just nonexudative AMD, 37 patients having just exudative AMD, and ten patients having both exudative and nonexudative AMD. The prevalence of just nonexudative AMD was $1.6 \%$ $(84 / 5,154)$ and the prevalence of exudative AMD was $0.91 \%$ $(47 / 5,154)$. Patients with both exudative and nonexudative AMD were included in the prevalence calculations for exudative AMD because having both diagnoses may represent progression from nonexudative AMD to exudative AMD. POAG patients and $\mathrm{PXG}$ patients had a higher prevalence of nonexudative AMD than CACG patients $(2.0 \%$ versus $0 \%$, $P=0.009$ and $2.9 \%$ versus $0 \%, P=0.004$, Fisher's exact test). There was no difference in mean age between POAG and PXG patients with nonexudative AMD (84.9 versus 86.3, $P>0.05, t$-test). There were no differences in the prevalence of exudative AMD according to type of glaucoma $(0 \%-1.5 \%$, $P>0.05$, Fisher's exact test).

Lastly, we examined blindness and low vision to determine if glaucoma patients with comorbid retinal disease have a higher prevalence of severe vision loss. Glaucoma patients with comorbid retinal disease had a higher prevalence of

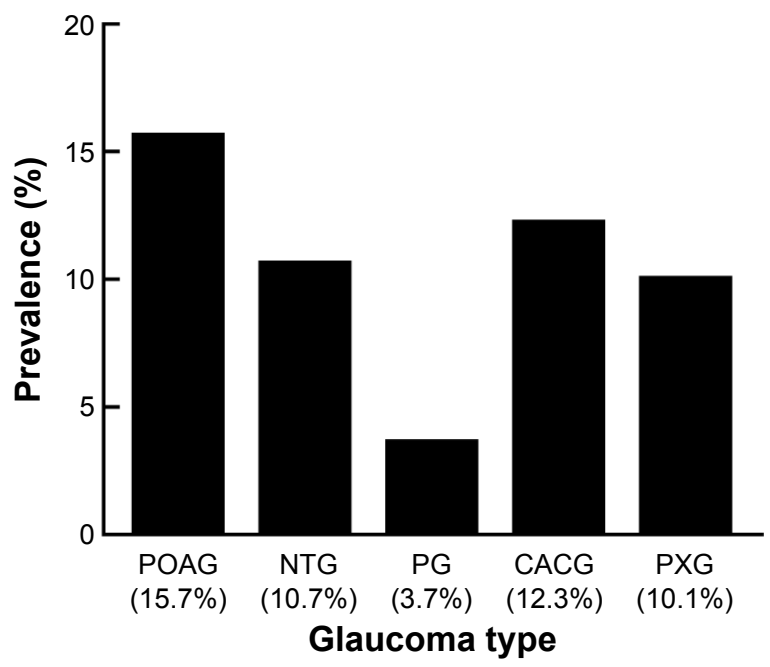

Figure I Prevalence of comorbid retinal disease by glaucoma type. POAG patients (15.7\%) had a higher prevalence of comorbid retinal disease than NTG (10.7\%), PXG (10.1\%), and PG (3.7\%) patients.

Note: $P<0.05, \chi^{2}$ test.

Abbreviations: POAG, primary open-angle glaucoma; NTG, low tension openangle glaucoma; PG, pigmentary open-angle glaucoma; CACG, chronic angle-closure glaucoma; PXG, pseudoexfoliation glaucoma. severe vision loss than those without retinal disease $(1.97 \%$ versus $1.02 \%, P=0.02, \chi^{2}$ test, Figure 3 ).

\section{Discussion}

The purpose of this study was to estimate the prevalence of retinal disease in a population of long-term glaucoma patients at a single academic medical center in the USA and to determine if there are associations between specific types of glaucoma and the most common comorbid retinal diseases. This was a billing-based study and not a chart review, so clinical and laboratory data such as IOP, refraction, body mass index, and glycated hemoglobin were not available. The strengths of the current study include the large number of glaucoma patients who all had long-term follow-up. We required a minimum of 2 years of follow-up to be included in the study, and $41.6 \%$ of patients were seen for at least 5 years. We utilized a dataset from a single, tertiary care medical center in order to capture patients who visited the same center for both retinal and glaucoma care although prevalence rates may reflect referral bias. Weaknesses of the current study include the retrospective design, reliance on ICD-9 codes without a chart review to confirm patient diagnoses, lack of a control group, and inclusion of patients with only five types of glaucoma. Patients who were seen for glaucoma at this institution and saw an outside doctor for any retinal disease were not captured in our analysis. Patients with CACG and narrow-angle glaucoma may be at higher risk for complications from dilation, and fundus examinations in these patients may be suboptimal. It is possible that the risk of dilation may have decreased the diagnosis of comorbid retinal disease in this glaucoma population. Further studies are needed to evaluate national prevalence rates among subtypes of glaucoma patients and to evaluate associations between POAG and specific retinal diseases compared with age-matched and sex-matched controls. Detailed chart reviews can be utilized to confirm the results of billing database studies and can also include clinical data such as IOP.

We selected POAG, NTG, CACG, pigmentary openangle glaucoma, and PXG because these were the adult 
Table 3 Patients with ICD-9 36 I diagnoses: retinal detachments and defects $(n=5 I)$

\begin{tabular}{ll}
\hline 361.0 & Retinal detachment with retinal defect \\
361 & Retinal detachment with retinal defect, unspecified \\
$361.01-0.04$ & Recent retinal detachment, partial \\
361.05 & Recent retinal detachment, total or subtotal \\
361.06 & Old retinal detachment, partial \\
361.07 & Old retinal detachment, total or subtotal \\
361.1 & Retinoschisis and retinal cysts \\
361.1 & Retinoschisis, unspecified \\
361.11 & Flat retinoschisis \\
361.12 & Bullous retinoschisis \\
361.13 & Primary retinal cysts \\
361.14 & Secondary retinal cysts \\
361.19 & Other retinoschisis and retinal cysts \\
361.2 & Serous retinal detachment \\
361.3 & Retinal defects without detachment \\
361.3 & Retinal defect, unspecified \\
361.31 & Round hole of retina without detachment \\
361.32 & Horseshoe tear of retina without detachment \\
361.33 & Multiple defects of retina without detachment \\
361.8 & Other forms of retinal detachment \\
361.81 & Traction detachment of retina \\
361.89 & Other forms of retinal detachment \\
$\mathbf{3 6 1 . 9}$ & Unspecified retinal detachment \\
& Total number of diagnoses \\
\hline A6 & 36 \\
\end{tabular}

Abbreviation: ICD-9, International Classification of Disease.

Table 4 Patients with ICD-9 362 diagnoses: other retinal disorders $(n=684)$

\begin{tabular}{|c|c|c|}
\hline 362.0 & Diabetic retinopathy & 277 \\
\hline 362.01 & Background diabetic retinopathy & 82 \\
\hline 362.02 & Proliferative diabetic retinopathy & 78 \\
\hline 362.03 & Nonproliferative diabetic retinopathy, NOS & 70 \\
\hline 362.04 & Mild nonproliferative diabetic retinopathy & 9 \\
\hline 362.05 & Moderate nonproliferative diabetic retinopathy & 2 \\
\hline 362.06 & Severe nonproliferative diabetic retinopathy & 5 \\
\hline 362.07 & Diabetic macular edema & 31 \\
\hline 362.1 & Other background retinopathy and retinal vascular changes & 16 \\
\hline 362.10 & Background retinopathy, unspecified & 1 \\
\hline 362.11 & Hypertensive retinopathy & 1 \\
\hline 362.12 & Exudative retinopathy & I \\
\hline 362.13 & Changes in vascular appearance of retina & I \\
\hline 362.14 & Retinal microaneurysms, NOS & I \\
\hline 362.15 & Retinal telangiectasia & 0 \\
\hline 362.16 & Retinal neovascularization, NOS & 11 \\
\hline 362.17 & Other intraretinal microvascular abnormalities & 0 \\
\hline 362.18 & Retinal vasculitis & 0 \\
\hline 362.2 & Other proliferative retinopathy & 3 \\
\hline 362.20 & Retinopathy of prematurity, unspecified & 0 \\
\hline 362.21 & Retrolental fibroplasia & 1 \\
\hline $362.22-362.27$ & Retinopathy of prematurity, stage $0-5$ & 0 \\
\hline 362.29 & Other nondiabetic proliferative retinopathy & 2 \\
\hline 362.3 & Retinal vascular occlusion & 140 \\
\hline 362.30 & Retinal vascular occlusion, unspecified & 1 \\
\hline 362.31 & Central retinal artery occlusion & 3 \\
\hline 362.32 & Retinal arterial branch occlusion & 4 \\
\hline 362.33 & Partial retinal arterial occlusion & 0 \\
\hline 362.34 & Transient retinal arterial occlusion & 0 \\
\hline 362.35 & Central retinal vein occlusion & 67 \\
\hline
\end{tabular}


Table 4 (Continued)

\begin{tabular}{|c|c|c|}
\hline 362.36 & Venous tributary (branch) occlusion & 65 \\
\hline 362.37 & Venous engorgement & 0 \\
\hline 362.4 & Separation of retinal layers & 10 \\
\hline 362.40 & Retinal layer separation, unspecified & 0 \\
\hline 362.41 & Central serous retinopathy & 5 \\
\hline 362.42 & Serous detachment of retinal pigment epithelium & 5 \\
\hline 362.43 & Hemorrhagic detachment of retinal pigment epithelium & 0 \\
\hline 362.5 & Degeneration of macula and posterior pole of retina & 456 \\
\hline 362.50 & Macular degeneration (senile), unspecified & 89 \\
\hline 362.51 & Nonexudative senile macular degeneration & 94 \\
\hline 362.52 & Exudative senile macular degeneration & 47 \\
\hline 362.53 & Cystoid macular degeneration & 125 \\
\hline 362.54 & Macular cyst, hole, or pseudohole & 26 \\
\hline 362.55 & Toxic maculopathy & 1 \\
\hline 362.56 & Macular puckering & 69 \\
\hline 362.57 & Drusen (degenerative) & 5 \\
\hline 362.6 & Peripheral retinal degenerations & 5 \\
\hline 362.60 & Peripheral retinal degeneration, unspecified & I \\
\hline 362.61 & Paving stone degeneration & 0 \\
\hline 362.62 & Microcystoid degeneration & 0 \\
\hline 362.63 & Lattice degeneration & 4 \\
\hline 362.64 & Senile reticular degeneration & 0 \\
\hline 362.65 & Secondary pigmentary degeneration & 0 \\
\hline 362.66 & Secondary vitreoretinal degeneration & 0 \\
\hline 362.7 & Hereditary retinal dystrophies & 12 \\
\hline 362.70 & Hereditary retinal dystrophy, unspecified & I \\
\hline 362.71 & Retinal dystrophy in systemic or cerebroretinal lipidoses & 0 \\
\hline 362.72 & Retinal dystrophy in other systemic disorders and syndromes & 0 \\
\hline 362.73 & Vitreoretinal dystrophies & 0 \\
\hline 362.74 & Pigmentary retinal dystrophy & 6 \\
\hline 362.75 & Other dystrophies primarily involving the sensory retina & 3 \\
\hline 362.76 & Dystrophies primarily involving the retinal pigment epithelium & 2 \\
\hline 362.77 & Dystrophies primarily involving Bruch's membrane & 0 \\
\hline 362.8 & Other retinal disorders & 17 \\
\hline 362.81 & Retinal hemorrhage & 0 \\
\hline 362.82 & Retinal exudates and deposits & 0 \\
\hline 362.83 & Retinal edema & 12 \\
\hline 362.84 & Retinal ischemia & 2 \\
\hline 362.85 & Retinal nerve fiber bundle defects & 0 \\
\hline 362.89 & Other retinal disorders & 3 \\
\hline \multirow[t]{2}{*}{362.9} & Unspecified retinal disorder & 0 \\
\hline & Total number of diagnoses & 936 \\
\hline
\end{tabular}

Abbreviations: ICD-9, International Classification of Disease; NOS, not otherwise specified.

Table 5 Patients with ICD-9 363 diagnoses: chorioretinal inflammation, scars and other disorders of the choroid $(n=I 7 I)$

\begin{tabular}{lll}
\hline 363.0 & Focal chorioretinitis and focal retinochoroiditis & $\mathbf{0}$ \\
$363.00-0.08$ & Focal retinitis, chorioretinitis, and retinochoroiditis & 0 \\
363.1 & Disseminated chorioretinitis and disseminated retinochoroiditis & I \\
363.10 & Disseminated chorioretinitis, unspecified & 0 \\
363.11 & Disseminated choroiditis and chorioretinitis, posterior pole & 0 \\
363.12 & Disseminated choroiditis and chorioretinitis, peripheral & I \\
363.13 & Disseminated choroiditis and chorioretinitis, generalized & 0 \\
363.14 & Disseminated retinitis and retinochoroiditis, metastatic & 0 \\
363.15 & Disseminated retinitis and retinochoroiditis, pigment epitheliopathy & I \\
362.2 & Other and unspecified forms of chorioretinitis and retinochoroiditis & 0 \\
363.20 & Chorioretinitis, unspecified & 0 \\
363.21 & Pars planitis &
\end{tabular}


Table 5 (Continued)

\begin{tabular}{|c|c|c|}
\hline 363.22 & Harada's disease & 1 \\
\hline 363.3 & Chorioretinal scars & II \\
\hline 363.30 & Chorioretinal scar, unspecified & 4 \\
\hline 363.31 & Solar retinopathy & 0 \\
\hline 363.32 & Other macular scars & 7 \\
\hline 363.33 & Other scars of posterior pole & 0 \\
\hline 363.34 & Peripheral scars & 0 \\
\hline 363.35 & Disseminated scars & 0 \\
\hline 363.4 & Choroidal degenerations & $\mathbf{I}$ \\
\hline 363.40 & Choroidal degeneration, unspecified & 0 \\
\hline 363.41 & Senile atrophy of choroid & 0 \\
\hline 363.42 & Diffuse secondary atrophy of choroid & 0 \\
\hline 363.43 & Angioid streaks of choroid & 1 \\
\hline 363.5 & Hereditary choroidal dystrophies & 0 \\
\hline $363.50-57$ & Hereditary choroidal dystrophy or atrophy & 0 \\
\hline 363.6 & Choroidal hemorrhage and rupture & 0 \\
\hline $363.6 I-63$ & Choroidal hemorrhage & 0 \\
\hline 363.7 & Choroidal detachment & 2 \\
\hline 363.70 & Choroidal detachment, unspecified & 2 \\
\hline 363.71 & Serous choroidal detachment & 0 \\
\hline 363.72 & Hemorrhagic choroidal detachment & 0 \\
\hline 363.8 & Other disorders of choroid & I \\
\hline \multirow[t]{2}{*}{363.9} & Unspecified disorder of choroid & 0 \\
\hline & Total number of diagnoses & 17 \\
\hline
\end{tabular}

Abbreviation: ICD-9, International Classification of Disease.

glaucoma patients most commonly seen at our academic medical center. We excluded neovascular glaucoma because of the well-established associations with proliferative diabetic retinopathy and ocular ischemic syndrome. ${ }^{22} \mathrm{We}$ identified patients who had at least three clinical examinations over the course of 2 years with the same glaucoma diagnosis. We chose these inclusion criteria in order to maximize the accuracy of glaucoma diagnosis, to identify a glaucoma population with multiple years of follow-up care, and to minimize the inclusion of glaucoma patients who were seen at this academic medical center for just a second opinion. Retina patients were required to have the same diagnosis associated with two visits of any type (clinic, procedure, or emergency room). The requirement

Table 6 Patients with ICD-9 379.2 diagnoses: disorders of the vitreous body $(n=43)$

\begin{tabular}{lll}
\hline 379.2 & Disorders of the vitreous body & 0 \\
379.21 & Vitreous degeneration & 19 \\
379.22 & Crystalline deposits in vitreous & 1 \\
379.23 & Vitreous hemorrhage & 12 \\
379.24 & Other vitreous opacities & 0 \\
379.25 & Vitreous membranes and strands & 0 \\
379.26 & Vitreous prolapse & 10 \\
379.27 & Vitreomacular adhesion & 0 \\
379.29 & Other disorders of the vitreous & 1 \\
& Total number of diagnoses & 43 \\
\hline
\end{tabular}

Abbreviation: ICD-9, International Classification of Disease. for at least two visits with the same retinal diagnosis may be expected to reduce false positive (inclusion) diagnostic inaccuracy arising from single coding errors or from evaluation visits found to be negative for a referral question. In addition, glaucoma diagnoses tend to be more consistent over time because patients with retinal disease may have disease progression and therefore have a change in diagnosis (eg, from nonproliferative diabetic retinopathy to proliferative diabetic retinopathy). We selected less stringent criteria for blindness and low vision (two visits with any ICD-9 code 369) because this diagnosis is relatively rare, and we were

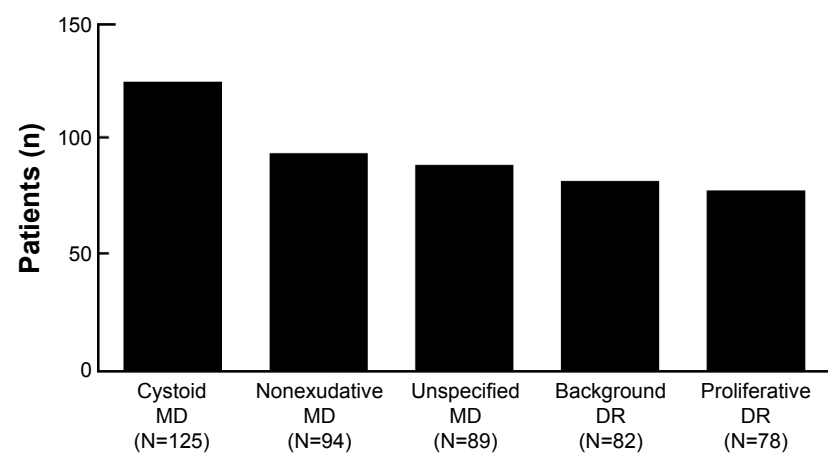

Comorbid retinal diagnoses

Figure 2 Most common comorbid retinal diagnoses. Of patients with glaucoma, the five most common retinal diagnoses were cystoid MD, nonexudative $M D$, unspecified MD, background DR, and proliferative DR.

Abbreviations: $\mathrm{DR}$, diabetic retinopathy; MD, macular degeneration. 


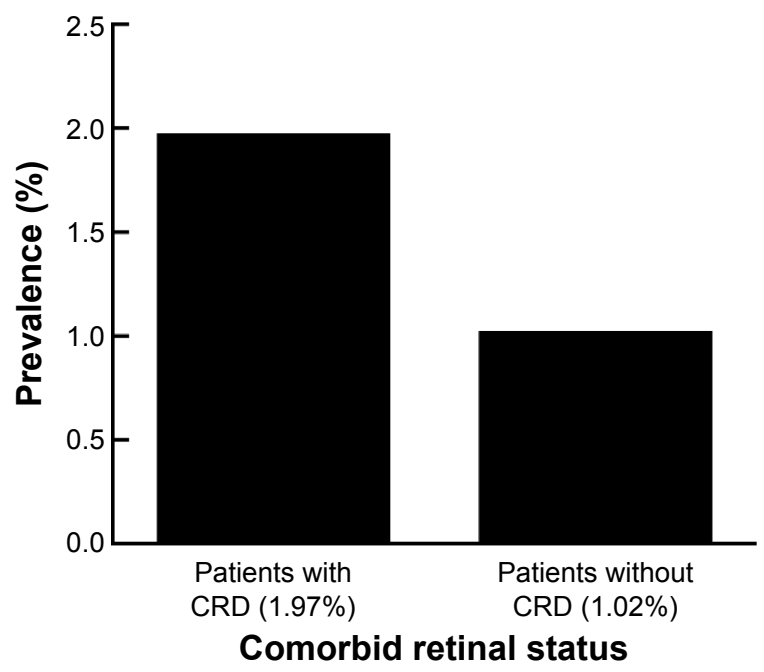

Figure 3 Prevalence of blindness and low vision. Glaucoma patients with comorbid retinal disease have higher rates of blindness and low vision than glaucoma patients without comorbid retinal disease (1.97\% versus $1.02 \%$ ).

Note: $P=0.02, \chi^{2}$ test.

Abbreviation: CRD, comorbid retinal disease.

concerned in this study with whether a patient develops severe vision loss and not the specific level of severe vision loss (eg, total, near-total, profound).

Previous studies have determined that patients with specific retinal diseases, retinal surgeries, and retinal procedures have an increased incidence of ocular hypertension and glaucoma; however, there are no publications to our knowledge that estimate the prevalence of retinal comorbidities in a population of glaucoma patients. We found significant rates of comorbid retinal disease (14.8\%) in this glaucoma population. Glaucoma patients with comorbid disease have a higher mean age than those without comorbid retinal disease. The higher mean age may be because increasing age is a risk factor for many retinal diseases. There was no association between sex and rates of comorbid retinal disease.

There are no previous studies on the rates of comorbid retinal disease in patients with glaucoma. Epidemiology studies, however, can provide information on rates of certain diseases in the general population. These studies are drawn from different populations and have different designs, so comparisons are limited. Rates of diabetic retinopathy in this study are consistent with epidemiologic studies in the ophthalmology literature. The results indicating that $3.4 \%$ of glaucoma patients have diabetic retinopathy and $3.9 \%$ of patients have either diabetic retinopathy or diabetes with ophthalmic manifestations are consistent with previous studies which estimated that the prevalence of diabetic retinopathy is $3.4 \%$ among Americans over 40 years of age. ${ }^{23}$ Thus, using historical controls derived from epidemiological studies, the prevalence of diabetic retinopathy appears to be no different in glaucoma patients than in community-based screening samples.

In contrast, rates of vascular occlusions in this study are higher than other prevalence estimates. We combined BRVO and CRVO for a combined prevalence of $2.4 \%$. Globally, the prevalence is estimated to be 3.77 per 1,000 for BRVO and 0.65 per 1,000 for $\mathrm{CRVO} .{ }^{24}$ Our higher prevalence rates may reflect referral bias or may demonstrate that glaucoma patients have a higher prevalence than the general public. Open-angle glaucoma has long been a recognized risk factor for both BRVO and CRVO. ${ }^{25,26}$ Ischemic CRVO can lead to neovascular glaucoma and CACG, but this study did not include neovascular glaucoma, and there were no statistically significant differences in rates of vein occlusions or vascular occlusions between the glaucoma types studied here. It is possible that CACG was underdiagnosed in our study if patients did not undergo gonioscopy. The small numbers of patients with a vascular occlusion also limited the statistical power of this study. Of all study patients, only three PXG, five NTG, eight CACG, and 112 POAG patients had a vascular occlusion.

On the other end of the spectrum, our results for the prevalence of macular degeneration in glaucoma patients are lower than prevalence rates from previous studies. A 2004 meta-analysis estimated the prevalence of AMD to be $6.12 \%$ for early AMD and 1.02\% for neovascular AMD, ${ }^{27}$ which is higher than our estimates of $2.5 \%$ for either exudative or nonexudative AMD, $1.6 \%$ for just nonexudative AMD, and 0.91\% for exudative AMD. In a 2008 study of Finnish patients with POAG and PXG, the prevalence of AMD was $4.0 \%{ }^{28}$ The definition of AMD is a key determinant of how prevalence rates are calculated, and the 2004 meta-analysis defined early AMD as having at least one drusen of $125 \mu \mathrm{m}$ or larger. ${ }^{27}$ The lower rates of AMD in this study may reflect the limitation of using ICD-9 codes without a chart review. For instance, the proportion of nonexudative AMD patients to exudative AMD patients in this study is $2.5 \%$ to $0.91 \%$, which differs from well-established estimates that nonexudative AMD represents $85 \%-90 \%$ of all AMD patients. Together these data may reflect an ascertainment bias where, in the absence of formal prospective study, drusen reflecting early AMD may not have been detected or recorded, or may not have led to referral to retina specialists more likely to code these diseases. Another explanation for the low rates of AMD is that this study population may not be generalizable to a communitybased epidemiological study. While we do not have race or ethnicity information on study participants, this academic medical center is located in a county that is $64.3 \%$ Hispanic or Latino according to the US Census Bureau. ${ }^{29}$ It has long been 
established that Caucasians have higher rates of AMD than African Americans, and a recent study suggests that Hispanics have lower rates of AMD than Caucasians. ${ }^{30}$ Finally, the lower rates of AMD could suggest a hypothesis that glaucoma may be a protective factor against the development of AMD, although this hypothesis would need considerable additional study as well as a plausible biological mechanism.

When examining the differences in prevalence of comorbid retinal disease between types of glaucoma, we also evaluated differences in mean age between glaucoma types, as age could be a confounder given that it is a known risk factor for both glaucoma and many retinal diseases such as AMD, atherosclerosis and retinal artery occlusive disease, retinal venous occlusive disease, and epiretinal membrane. ${ }^{31}$ Of all the patients with comorbid retinal disease, $\mathrm{PXG}$ patients were older than those with POAG, NTG, and CACG, and there were no other differences between glaucoma types. For instance, POAG patients with comorbid retinal disease were not older than patients with other types of glaucoma.

The nearly twofold increased risk of severe vision loss in patients with comorbid retinal disease highlights the need for physicians to include a careful retinal examination, and to consider multiple etiologies if visual field results or optic nerve assessments do not correlate with the level of vision loss. A multidisciplinary, team-based approach to patient care with more frequent monitoring is clearly indicated for patients at increased risk for vision loss. Understanding the prevalence of comorbid retinal disease in patients with certain types of glaucoma may lead to increased awareness among eye care professionals. Further studies are needed to evaluate the care of glaucoma patients with comorbid retinal disease.

\section{Acknowledgment}

The authors gratefully acknowledge the National Eye Institute (P30s EY022589 and EY014801) and an unrestricted grant from Research to Prevent Blindness Inc.

\section{Disclosure}

JFG: Pfizer, Inc. (Ownership). The preliminary data from this study were presented as a poster at the Association for Research in Vision and Ophthalmology national meeting at Orlando, FL, USA, in May 2014. The authors report no conflicts of interest in this work.

\section{References}

1. Ritch R, Shields M. The Secondary Glaucomas. St Louis, MO: CV Mosby; 1982.

2. Panek WC, Holland GN, Lee DA, Christensen RE. Glaucoma in patients with uveitis. Br J Ophthalmol. 1990;74(4):223-227.
3. Neri P, Azuara-Blanco A, Forrester JV. Incidence of glaucoma in patients with uveitis. J Glaucoma. 2004;13(6):461-465.

4. Merayo-Lloves J, Power WJ, Rodriguez A, Pedroza-Seres M, Foster CS Secondary glaucoma in patients with uveitis. Ophthalmologica. 1999;213(5):300-304.

5. Siddique SS, Suelves AM, Baheti U, Foster CS. Glaucoma and uveitis. Surv Ophthalmol. 2013;58(1):1-10.

6. Elgin U, Berker N, Batman A. Incidence of secondary glaucoma in Behçet disease. J Glaucoma. 2004;13(6):441-444.

7. Badeeb $\mathrm{O}$, Trope $\mathrm{G}$, Musarella M. Primary angle closure glaucoma and retinitis pigmentosa. Acta Ophthalmol. 1993;71(6):727-732.

8. Phelps CD, Burton TC. Glaucoma and retinal detachment. Arch Ophthalmol. 1977;95(3):418-422.

9. Kocabora MS, Yilmazli C, Taskapili M, Gulkilik G, Durmaz S. Development of ocular hypertension and persistent glaucoma after intravitreal injection of triamcinolone. Clin Ophthalmol. 2008;2(1):167-171.

10. Jones R 3rd, Rhee DJ. Corticosteroid-induced ocular hypertension and glaucoma: a brief review and update of the literature. Curr Opin Ophthalmol. 2006;17(2):163-167.

11. Tsai JC, Lee MB, Wudunn D, Dacey MP, Choi JC, Minckler DS. Incidence of acute intraocular pressure elevation after panretinal photocoagulation. J Glaucoma. 1995;4(1):45-48.

12. Aiello LM. Perspectives on diabetic retinopathy. Am JOphthalmol.2003; 136(1):122-135.

13. Blondeau P, Pavan PR, Phelps CD. Acute pressure elevation following panretinal photocoagulation. Arch Ophthalmol. 1981;99(7): 1239-1241.

14. Hoang QV, Mendonca LS, Della Torre KE, Jung JJ, Tsuang AJ, Freund KB. Effect on intraocular pressure in patients receiving unilateral intravitreal anti-vascular endothelial growth factor injections. Ophthalmology. 2012;119(2):321-326.

15. Tseng JJ, Vance SK, Della Torre KE, et al. Sustained increased intraocular pressure related to intravitreal antivascular endothelial growth factor therapy for neovascular age-related macular degeneration. J Glaucoma. 2012;21(4):241-247.

16. Anderson NG, Fineman MS, Brown GC. Incidence of intraocular pressure spike and other adverse events after vitreoretinal surgery. Ophthalmology. 2006;113(1):42-47.

17. Desai UR, Alhalel AA, Schiffman RM, Campen TJ, Sundar G, Muhich A. Intraocular pressure elevation after simple pars plana vitrectomy. Ophthalmology. 1997;104(5):781-786.

18. Muether PS, Hoerster R, Kirchhof B, Fauser S. Course of intraocular pressure after vitreoretinal surgery: is early postoperative intraocular pressure elevation predictable? Retina. 2011;31(8):1545-1552.

19. Honavar SG, Goyal M, Majji AB, Sen PK, Naduvilath T, Dandona L. Glaucoma after pars plana vitrectomy and silicone oil injection for complicated retinal detachments. Ophthalmology. 1999;106(1):169-176.

20. Koreen L, Yoshida N, Escariao P, et al. Incidence of, risk factors for, and combined mechanism of late-onset open-angle glaucoma after vitrectomy. Retina. 2012;32(1):160-167.

21. Chang S. LXII Edward Jackson lecture: open angle glaucoma after vitrectomy. Am J Ophthalmol. 2006;141(6):1033-1043.

22. Shazly TA, Latina MA. Neovascular glaucoma: etiology, diagnosis and prognosis. Semin Ophthalmol. 2009;24(2):113-121.

23. Kempen JH, O'Colmain BJ, Leske MC, et al. The prevalence of diabetic retinopathy among adults in the United States. Arch Ophthalmol. 2004;122(4):552-563.

24. Rogers S, McIntosh RL, Cheung N, et al. The prevalence of retinal vein occlusion: pooled data from population studies from the United States, Europe, Asia, and Australia. Ophthalmology. 2010;117(2): 313-319.e1.

25. [No authors listed]. Risk factors for central retinal vein occlusion. The Eye Disease Case-Control Study Group. Arch Ophthalmol. 1996; 114(5):545-554.

26. [No authors listed]. Risk factors for branch retinal vein occlusion. The Eye Disease Case-control Study Group. Am J Ophthalmol. 1993; 116(3):286-296. 
27. Friedman DS, O’Colmain BJ, Munoz B, et al. Prevalence of age-related macular degeneration in the United States. Arch Ophthalmol. 2004; 122(4):564-572.

28. Tarkkanen A, Reunanen A, Kivela T. Frequency of age-related macular degeneration among patients with primary chronic open-angle glaucoma and exfoliation glaucoma. Acta Ophthalmol. 2008;86(6):697-698.

29. US Census Bureau. State and County QuickFacts. Miami-Dade, Florida. Available from: http://quickfacts.census.gov/qfd/states/12/12086.html. Accessed April 28, 2014.
30. Vanderbeek BL, Zacks DN, Talwar N, Nan B, Musch DC, Stein JD. Racial differences in age-related macular degeneration rates in the United States: a longitudinal analysis of a managed care network. Am J Ophthalmol. 2011;152(2):273-282.e3.

31. Kanski JJ. Clinical Ophthalmology: A Systematic Approach. 6th ed. Philadelphia, PA: Elsevier; 2007.

\section{Publish your work in this journal}

Clinical Ophthalmology is an international, peer-reviewed journal covering all subspecialties within ophthalmology. Key topics include: Optometry; Visual science; Pharmacology and drug therapy in eye diseases; Basic Sciences; Primary and Secondary eye care; Patient Safety and Quality of Care Improvements. This journal is indexed on

\section{Dovepress}

PubMed Central and CAS, and is the official journal of The Society of Clinical Ophthalmology (SCO). The manuscript management system is completely online and includes a very quick and fair peer-review system, which is all easy to use. Visit http://www.dovepress.com/ testimonials.php to read real quotes from published authors. 